\title{
RNASET2 silencing affects miRNAs and target gene expression pattern in a human ovarian cancer cell model
}

\author{
GIOVANNA TURCONI ${ }^{1 *, \#}$, DEBORA SCALDAFERRI ${ }^{2 *}$, MARCO FABBRI $^{3}$, LAURA MONTI ${ }^{2}$, MARTA LUALDI $^{1,5}$, \\ EDOARDO PEDRINI $^{2}$, LAURA GRIBALDO ${ }^{4}$, ROBERTO TARAMELLI $^{2 * *}$ and FRANCESCO ACQUATI ${ }^{2 * *}$ \\ Departments of ${ }^{1}$ Theoretical and Applied Sciences, ${ }^{2}$ Biotechnology and Life Sciences, and \\ ${ }^{3}$ Clinical and Experimental Medicine, University of Insubria, Varese; ${ }^{4}$ JRC Directorate F - Health, Consumers \\ and Reference Materials, Chemical Safety and Alternative Methods Unit, Ispra, Varese (VA), Italy
}

Received July 29, 2016; Accepted November 7, 2016

DOI: $10.3892 /$ ijo.2016.3763

\begin{abstract}
Ribonucleases (RNases) are hydrolytic enzymes endowed with the ability to either process or degrade ribonucleic acids. Among the many biological functions assigned to RNases, a growing attention has been recently devoted to the control of cancer growth, in the attempt to bring novel therapeutic approaches to clinical oncology. Indeed, several enzymes belonging to different ribonuclease families have been reported in the last decade to display a marked oncosuppressive activity in a wide range of experimental models. The human RNASET2 gene, the only member of the highly conserved $\mathrm{T} 2 / \mathrm{Rh} / \mathrm{S}$ family of endoribonucleolytic enzymes described in our species, has been shown to display oncosuppressive roles in both in vitro and in vivo models representing several human malignancies. In the present study, we extend previous findings obtained in ovarian cancer models to shed further light on the cell-autonomous roles played by this gene in the context of its oncosuppresive role and to show that RNASET2 silencing can significantly affect the transcriptional output in one of the most thoroughly investigated human ovarian cancer cell lines. Moreover, we report for the first time that RNASET2-mediated changes in the cell transcriptome are in part mediated by its apparent ability to affect the cell's microRNA expression pattern.
\end{abstract}

Correspondence to: Professor Francesco Acquati, Department of Theoretical and Applied Sciences, University of Insubria, via $\mathrm{JH}$ Dunant 3, I-21100 Varese, Italy

E-mail: francesco.acquati@uninsubria.it

Present address: ${ }^{5}$ Department of Science and High Technology University of Insubria, Varese, Italy

*Contributed equally; ** Joint senior authorship

${ }^{\#}$ Deceased

Key words: T2 RNase, tumor suppression, miRNAs, ovarian cancer, tumor microenvironment, P-bodies

\section{Introduction}

Ovarian cancer represents the fifth most common malignancy in women and it is the leading cause of cancer deaths from gynecological cancer in the western societies (1). The poor survival from epithelial ovarian cancer that is still reported nowadays is largely attributed to the high percentage of patients that are diagnosed at an advanced stage and that often develop resistance to combined chemotherapy approaches (2). In light of this imperfect state of clinical management, a better understanding of the molecular bases of ovarian cancer, coupled with improved screening tools, is urgently required. In this context, an increasing number of independent studies have recently suggested that microRNAs (miRNAs) might play a crucial role in ovarian cancer pathogenesis (3-5).

Indeed, several miRNA profiling reports have identified a consensus of aberrantly expressed miRNAs in both ovarian cancer-derived tumor samples and cell lines (3), suggesting that these small RNAs might be functionally involved in ovarian cancer development and progression. Moreover, both gain-offunction and loss-of-function assays have further validated the role played by several miRNAs in ovarian cancer pathogenesis (4).

MicroRNAs represent a large family of small endogenous non-coding RNAs endowed with the ability to regulate gene expression post-transcriptionally (6). The widely recognized role assigned to miRNAs in cancer biology is a natural consequence of their involvement in several biological processes that are closely linked to cancer development and progression, such as cell proliferation, differentiation and apoptosis (7). Indeed, the list of genes that have been reported to be regulated by miRNAs includes several examples of established tumor suppressor genes and oncogenes, such as BRCA1, BRCA2, PTEN, KIT, RAS, E2F3, MET and MYCN, to name a few (8). Therefore, substantial experimental evidence has been gathered in support of the hypothesis that derangement of a complex cross-regulatory network between miRNAs and transcripts from cancer-related genes might represent a critical event in the development and progression of several cancer types. However, due to the widely acknowledged complexity and heterogeneity of each cancer type, the current knowledge 
of the miRNA regulatory networks involved in cancer development is still far from providing an exhaustive picture.

The RNASET2 gene encodes a highly conserved extracellular ribonuclease belonging to the $\mathrm{T} 2 / \mathrm{Rh} / \mathrm{S}$ family of endoribonucleolytic enzymes and it has long been ranked as an ovarian cancer-related tumor suppressor gene (9). Moreover, several independent studies have consistently reported the oncosuppressive role of RNASET2 (both in vitro and in vivo) in a broader range of cancer types, such as malignant melanoma and colorectal cancer $(10,11)$. In the context of ovarian cancer, our group has recently described a marked non-cell autonomous oncosuppressive function for RNASET2. Indeed, in vivo xenograft studies in immunocompromised mice showed that RNASET2-mediated tumor suppression was associated with a significant recruitment of cancer-suppressive macrophages within the tumor mass in two independent human ovarian cancer-derived cell lines $(12,13)$. Strikingly, the catalytic activity of this enzyme was found to be dispensable for its non-cell autonomous oncosuppressive role, suggesting that RNASET2 might represent a multitasking or 'moonlightning' protein. In agreement with this speculation, we reported more recently a clear pleiotropic mode of action for the RNASET2 gene, whereby gene knock-down approaches coupled with a panel of in vitro assays unveiled the ability of RNASET2 to affect several cancer related-parameters in a cell-autonomous manner (14). Of note, these data were reported for the same experimental model (the human OVCAR3 ovarian cancer cell line) where previous in vivo studies had shown the occurrence of a non-cell autonomous oncosuppresive role for this gene (13). However, the genes and signaling networks by which RNASET2 carries out such cell-autonomous oncosuppressive role are largely unknown.

In an attempt to address this issue, we report here gene profiling data on RNASET2-silenced OVCAR3 cells and show that RNASET2 expression levels affects the cell transcriptome at both mRNAs and miRNAs levels. Moreover, our data suggest that the observed changes in the expression levels of some mRNAs represent a likely consequence of RNASET2mediated regulation of a handful of miRNAs that can directly target these transcripts.

\section{Materials and methods}

Cell lines and clones. Human ovarian cancer-derived OVCAR3 cell clones, stably transfected with empty $\mathrm{pSicoR}$ vector, control scrambled shRNAs and two RNASET2-targeting shRNAs were previously described (13). OVCAR3, Hey3Met2 and HeLa cells were cultured in DMEM-F12 medium (SigmaAldrich, St. Louis, MO, USA) with $10 \%$ fetal bovine serum (FBS; Sigma-Aldrich) and 1\% glutamine (Sigma-Aldrich).

RNA extraction. Total RNA was purified from OVCAR3 cells using the RNeasy Plus kit (Qiagen, Venlo, Limburg, The Netherlands). RNA was quantified using an ND-1000 UV-Vis spectrophotometer (Thermo Fisher Scientific, Wilmington, DE, USA), and the integrity of the RNA was assessed with the Agilent 2100 Bioanalyzer (Agilent Technologies, Inc., Santa Clara, CA, USA) according to the manufacturer's instructions. All of the RNA samples used in this study exhibited a 260/280 ratio above 1.9 and an RNA integrity number (RIN) above 9.0.
Microarray expression profiling. Microarray hybridization assays included two biological replicates per treatment. All sample-labelling, hybridization, washing, and scanning steps were carried out according to the manufacturer's specifications. Briefly, Cy3-labelled cRNA was generated from $50 \mathrm{ng}$ input total RNA using the One Color Quick Amp Labelling kit (Agilent Technologies). For every sample, 600 ng cRNA from each labelling reaction (with a specific activity above 9.0) was hybridized using the Gene Expression Hybridization kit (Agilent Technologies) to the Agilent Whole Human Genome Oligo Microarray (Agilent Technologies). After hybridization, the slides were washed and then scanned with the Agilent G2565BA microarray scanner (Agilent Technologies). The fluorescence intensities of the scanned images were extracted and pre-processed using Agilent Feature Extraction software (10.7.3.1).

Gene expression data analysis. Quality control and array normalization were performed in the R statistical environment using the Agi4x44PreProcess (v.1.18.0) package, which was downloaded from the Bioconductor website (15). The normalization and filtering steps were based on those described in the Agi4x44PreProcess reference manual. Briefly, the Agi4x44PreProcess options were set to use the Mean signal and the BG Median signal as foreground and background signals, respectively. The data were normalized between arrays using the quantile method. Genes with a fold change $>1 \log _{2}$ were designated as modulated. All of the above computations were conducted using the $\mathrm{R}$ statistical programming environment. The expression analysis systematic explorer (EASE) biological theme analysis of the regulated genes was conducted online using DAVID (16).

MicroRNA expression profiling. RNA was extracted using the mirVana kit (Ambion, Inc., Carlsbad, CA, USA) according to the manufacturer's instructions. Concentration and quality of RNA samples were determined with NanoDrop (Thermo Fisher Scientific). Total RNA was reverse transcribed with TaqMan MicroRNA reverse transcription kit using Megaplex RT Primers (Applied Biosystems, Foster City, CA, USA). Real-time PCR reactions were carried out on preconfigured microfluidic cards (TaqMan Array MicroRNA Cards, set A, V2.2 and set B, V3; Applied Biosystems) allowing the detection of $\sim 754$ unique specific assays and 4 candidate endogenous control assays. Two biological replicates for control and two for RNASET2-silenced cells were carried out. Experimental data were then analyzed by SDS 2.3 software (Applied Biosystems) and the relative expression values were calculated using U6 miRNA as endogenous control. miRNAs with a threshold cycle $<33$ that showed a $\log _{2}$-fold change greater than one in RNASET2-silenced samples compared to control samples were considered as induced. To derive a list of predicted target genes for miRNAs of interest, the selected miRNAs were uploaded into the miRecords database (http://miRecords.umn.edu/miRecords), in which the relationship between target genes and miRNAs are predicted by 11 different algorithms. The genes were identified as target when predicted by at least 5 algorithms. The predicted targets of the downregulated miRNAs were then crossed with the genes identified as upregulated by the microarray analysis. 
Real-time PCR analysis. Total RNA extracted from OVCAR3 cells was reverse transcribed with random primers using the 'High capacity cDNA reverse transcription kit' (Life Technologies). Primers for real-time qPCR were selected within the same region of the mRNA used to design the probes for microarray experiments. The sequences of the primers used for qPCR are as follows: 5'-AACTCCAGCAACTTCTT CTCCATCC-3' and 5'-AGAGAGGCCAGCTCAAGAGAA AC-3' for FOSL1; 5'-TCCCAGGACATGGCGAGGAGTA-3' and 5'-TCACTGGGAACCCGCAGGAAG-3' for NOTCH3; 5'-GTTCACTGTGTTTCTGCCGCTGTC-3' and 5'-CAGTTC TACATCCATGCCCAAGAAG-3' for DDIT4L; 5'-GAGTTC AGGCCTCTGGGATCAAC-3' and 5'-CGTTATCCTCCAA CCGGGCACAATA-3' for NFIB; 5'-TAGCTGCACAAGAAG CAAGAACCAG-3' and 5'-GCTGCCACCATGCACGAGG TTT-3' for PDE1A; 5'-GTAATGCTGCACCTCCCTCTC CT-3' and 5'-AACAAGCGTCTGGATCTCTGCAGG-3' for $L F N G$; 5'-CGCGAGAAGATGACCCAGAT-3' and 5'-ACAG CCTGGATAGCAACGTACA-3' for $\beta$-actin.

Real-time RT-PCR reactions were performed on ABI PRISM 7000 (Applied Biosystems) in triplicate in a $25 \mu 1$ volume containing target cDNA ( $25 \mathrm{ng}$ ), $40 \mathrm{nM}$ primers, $12.5 \mu 1$ of Power SYBR-Green Master Mix and water. Samples were denatured at $95^{\circ} \mathrm{C}$ for $15 \mathrm{sec}$ and annealed/extended at $60^{\circ} \mathrm{C}$ for $1 \mathrm{~min}$, for 40 cycles. Fluorescent signals generated during PCR amplification were monitored and analyzed with ABI PRISM 7000 SDS software (Applied Biosystems). Comparison of the amount of each gene transcript among different samples was made by using $\beta$-actin as a reference. The amount of target RNA, normalized to the endogenous reference gene, was calculated by means of the difference in threshold cycle parameter $(\Delta \Delta \mathrm{Cq})(17)$. The fold change in gene expression was calculated using this method and considering OVCAR3 parental cell line with a level of expression equal to one.

Cloning of 3'UTR regions from candidate target genes in pmirGLO. To test whether candidate target genes were regulated by the corresponding miRNAs, the 3'UTR regions of these genes were cloned in pmirGLO reporter vector (Promega). The selected regions were amplified with RT-PCR with specific primers, XhoI digested and ligated to the plasmid vector.

Cell transfection and reporter gene expression assays. pmirGLO recombinant constructs carrying the 3'UTR regions of candidate genes and the corresponding miRNAs were co-transfected in target cells with Lipofectamine 2000 reagent (Invitrogen, Carlsbad, CA, USA) following the manufacturer's instructions. A total of $100 \mathrm{ng}$ of vector and $1 \mathrm{pmol}$ of the corresponding miRNA were transfected in a $100 \mu \mathrm{l}$ final volume in 96-wells white plates with clear bottom. Twelve hours after the transfection, the medium was completely replaced and each well was filled with $75 \mu \mathrm{l}$ of fresh medium. Firefly and Renilla luciferases were activated using the Dual-Glo Luciferase assay system (Promega, Fitchburg, WI, USA) following the manufacturer's instructions and a luminometer was used to detect luminescence.

Fluorescence staining and cell size analysis of OVCAR3 cells. OVCAR3 cells were grown on coverslips for $24 \mathrm{~h}$, then processed for fluorescent staining of the actin cytoskeleton. Briefly, cells were fixed in 3\% paraformaldehyde, washed extensively in phosphate-buffered saline (PBS) and permeabilized with $0.1 \%$ Triton X-100 in PBS. After blocking (3\% BSA in PBS), cells were stained with $5 \mu \mathrm{g} / \mathrm{ml}$ PhalloidinTRITC (Phalloidin tetramethylrhodamine B isothiocyanate conjugated; Sigma-Aldrich). Coverslips were mounted on microscope slides and images were taken using a Leica TCS SP8 X confocal laser scanning microscope. Cell size analysis was performed using the Analyze Particle tool of ImageJ software.

Western blot analysis. Cultured OVCAR3 cells were mechanically scraped in PBS + $5 \mathrm{mM}$ EDTA and resuspended in lysis buffer $(0.5 \%$ Igepal, $0.5 \%$ Triton X-100 in PBS $+5 \mathrm{mM}$ EDTA) supplemented with a protease inhibitors and a phosphatase inhibitor cocktail. For SDS-PAGE analysis, $100 \mu \mathrm{g}$ of intracellular lysates were loaded for each gel lane. Immunoblot analysis was performed using standard procedures, using selected primary antibodies [rabbit anti-RNASET2 polyclonal antibody, rabbit anti-S6K1 (phospho T229) polyclonal antibody or mouse anti- $\alpha$-tubulin monoclonal antibody] followed by the appropriate horseradish peroxidase-conjugated antimouse or -rabbit IgG secondary antibody. Membranes were then processed with a chemiluminescence assay (SuperSignal West Dura; Thermo Fisher Scientific). The anti-S6K1 antibody was kindly gifted by Dr Nadia Zaffaroni (IRCCS Foundation, Milan, Italy).

Statistical analysis. Statistical analysis was performed using two-tailed Student's t-test, assuming $\mathrm{P}<0.05$ as a threshold value to discard the null hypothesis.

\section{Results}

Since a cell-autonomous oncosuppressive role for RNASET2 was previously established in a human ovarian cancer-derived cell line (OVCAR3) in which endogenous expression of this gene was silenced by RNA interference (13), we decided to exploit this experimental model to define genes and signaling networks involved in RNASET2-mediated tumor suppression.

In a previous study, we addressed the intracellular distribution pattern of the RNASET2 protein in order to define the putative sites where its function is carried out. In that study, a partial re-localization of the RNASET2 protein in processing bodies (P-bodies) was observed in OVCAR3 cells under stress condition (18). Moreover, the number of intracellular P-bodies turned out to be affected in RNASET2-silenced OVCAR3 cells (18), thus, suggesting a putative role for this protein in P-bodies function or assembly. Since they represent intracellular sites where RNA turnover takes place (19), the detection of RNASET2 in P-bodies led us to ask whether this enzyme plays a critical role in these subcellular structures. Of note, P-bodies represent sites where short-interfering RNAs (siRNAs) and microRNAs (miRNAs) carry out mRNA decay and/or translational repression (19). This fact, coupled to the established role for several miRNAs in the regulation of a wide range of cancer-related genes, prompted us to investigate the putative effects of RNASET2 on both miRNAs and mRNA transcriptional profiles. 
Table I. Differentially expressed microRNAs.

\begin{tabular}{lc}
\hline MicroRNA & Fold change \\
\hline hsa-miR-1227 & -3.3 \\
hsa-miR-200c & -2.2 \\
hsa-miR-577 & -1.9 \\
hsa-miR-183\# & -1.8 \\
hsa-miR-23b & -1.7 \\
hsa-miR-141 & -1.7 \\
hsa-miR-766 & -1.5 \\
hsa-miR-193b & -1.5 \\
hsa-miR-628-5p & -1.4 \\
hsa-miR-183 & -1.3 \\
hsa-miR-24 & -1.3 \\
hsa-miR-1260 & -1.2 \\
hsa-miR-454\# & -1.2 \\
hsa-miR-301a & -1.1 \\
hsa-miR-342-3p & -1.0 \\
hsa-miR-1233 & 1.1 \\
hsa-miR-21 & 1.1 \\
hsa-miR-935 & 1.5 \\
hsa-miR-20b & 1.9 \\
hsa-miR-449a & 3.6 \\
hsa-miR-206 & 3.9 \\
\hline
\end{tabular}

The table shows the microRNAs that were determined to be regulated with a fold change $>1$ when compared with the RNASET2-silenced OVCAR3 cells. The reported fold-changes express the difference of the mean log of the parental cells and the mean log of the RNASET2silenced cells. Fold change values shown in the second column are expressed as $\log _{2}$ values.

To this end, the gene expression profiles of parental (wt), RNASET2-silenced (shT2) and scrambled short-hairpin RNA (scrb)-transfected OVCAR3 cell clones were assessed by means of both quantitative PCR and microarray hybridization assays. As a first task, we assessed the expression profile of miRNAs by qPCR on a TaqMan array platform. From this assay, a few miRNAs turned out to be downregulated in RNASET2-silenced clones (15 miRNAs), whereas the others were upregulated (6 miRNAs) (Table I).

Based on these results, we decided to extend our transcriptome investigation on RNASET2-silenced and control OVCAR3 cells by addressing their mRNA expression profile by microarray hybridization. A preliminary analysis of the mRNA expression profile from scrb-transfected OVCAR3 cell clones showed that the expression of a few mRNAs was unexpectedly affected with respect to the parental OVCAR3 cells, likely due to the occurrence of unpredicted off-target effects from the scrambled shRNA vector used. We therefore decided to remove from further analysis those genes identified as differentially expressed (2-fold regulation) when (scrb)-transfected OVCAR3 were compared to parental (wt) OVCAR3 cells. Genes that were upregulated or downregulated in shT2 but not in scrb clones with respect to wt OVCAR3 cells were considered as interesting targets.

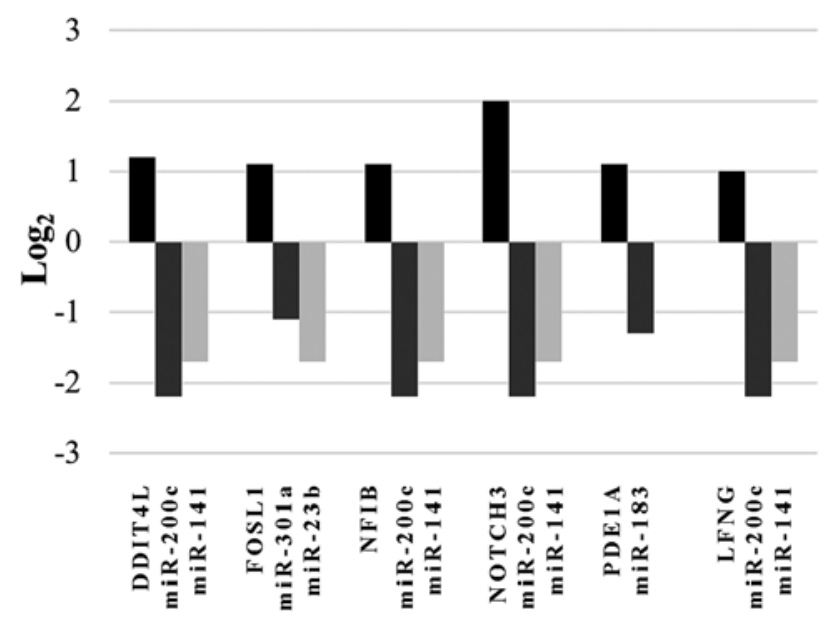

Figure 1. Inversely correlated expression of miRNAs and putative target genes. The graph shows fold changes in gene expression for miRNAs/target gene pairs in RNASET2-silenced OVCAR3 clones compared to control clones. A value of 1 means a fold change equal to 2 .

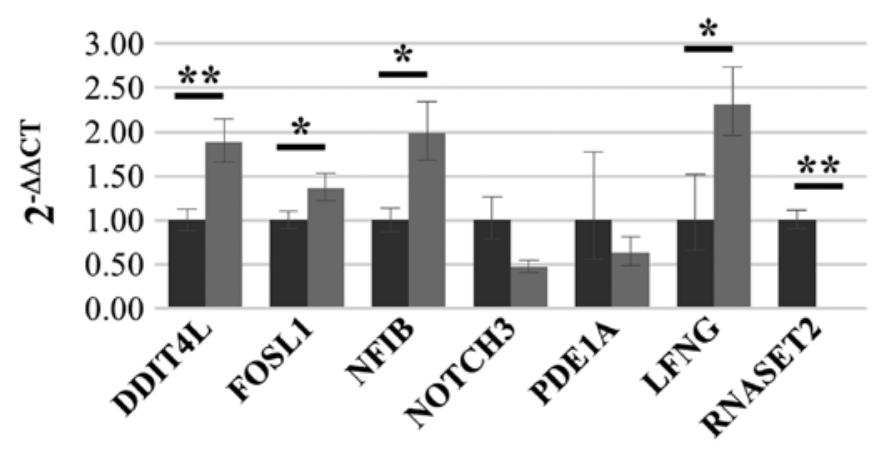

nOVCAR3 Parental cells OOVCAR3 RNASET2-silenced cells

Figure 2. Validation of microarray expression data for a panel of selected genes by real-time RT-PCR. qPCR assays were performed for each gene using the same RNA samples from the cells clones used for microarray hybridization assays. Error bars represent standard deviation from the mean. Statistical analysis was performed using two-tailed Student's t-test. ${ }^{*} \mathrm{P}<0.05$; ${ }^{* *} \mathrm{P}<0.01$.

A total of 299 mRNAs were found to show a 2-fold or greater change in expression level in RNASET2-silenced OVCAR3 cells when compared to wt cells. Among these genes, 184 were downregulated, whereas 115 were upregulated (the gene list is available on request). Significantly, cross-checking of the two sets of expression profiling data (miRNAs vs. mRNAs) showed that, for a few miRNAs that turned out to be downregulated in RNASET2-silenced clones, some of their putative target mRNAs were found to be upregulated in the subsequent gene expression profiling assay. In particular, 6 potential target mRNAs for some of these downregulated miRNAs turned out to be upregulated in RNASET2-silenced cells: $L F N G, F O S L 1$, $N F I B, D D I T 4 L, N O T C H 3$ and PDE1A. The inversely correlated expression pattern between these mRNAs and one or more of the selected miRNAs is shown in Fig. 1. Thus, these upregulated transcripts might represent potential targets for regulation by specific miRNAs whose expression is affected by RNASET2. Of note, annotation data from all 6 upregulated mRNAs showed a role for the corresponding genes in 
Table II. List of the genes selected.

\begin{tabular}{lllll}
\hline Gene ID and description & Functions & Cancer-related roles & Location \\
\hline
\end{tabular}

$N F I B$

(Nuclear factor I/B)

NOTCH3 (Notch3)

DDIT4L

(DNA-damage-inducible transcript 4-like)

LFNG

(LFNG O-fucosylpeptide 3-beta-N-acetylgluco-

saminyltransferase)

FOSL1

(FOS-like antigen 1)

PDE1A

(Phosphodiesterase 1A, calmodulin-dependent)
FOS proteins have been implicated as regulators of cell proliferation, differentiation, and transformation

Binds the palindromic sequence 5'-TTGGCNNNNNGCCAA-3' in viral and cellular promoters and in the origin of replication of adenovirus type 2 .

Functions as a receptor for membrane-bound ligands Jagged 1, Jagged 2 and Delta 1 to regulate cell-fate determination. Affects the implementation of differentiation, proliferation and apoptotic programs

Inhibits cell growth by regulating the TOR signaling pathway.

Regulator of NOTCH signalling

Cyclic nucleotide phosphodiesterase with a dual-specificity for the second messengers cAMP and cGMP, which are key regulators of many important physiological processes.
The gene is rearreanged in several cancer types and overexpressed in triple-negative breast cancer and small cell lung cancer.

Partner with MYB oncogene in cancer-associated translocations

Involved in proliferation of ERBB2-negative breast cancer cells. Overexpressed in serous ovarian carcinoma and non-small cell lung cancer.

Disregulated in breast cancer stromal tissue

4q23 miR-200c, miR-141

19p13.2 miR-200c, miR-141 -p13.1

Downregulated in basal-like breast cancer.

7p22.3 miR-200c, miR-141

Induces pro-tumoral M2

macrophage polarization pattern

11q13 miR-301a, miR-23b

Decreased PDE1A expression induces growth inhibition, cell cycle arrest and apoptosis in Jurkat cells

$$
2 \mathrm{q} 32.1
$$

miR-183 9p24.1 miR-200c, miR-141

\section{.}



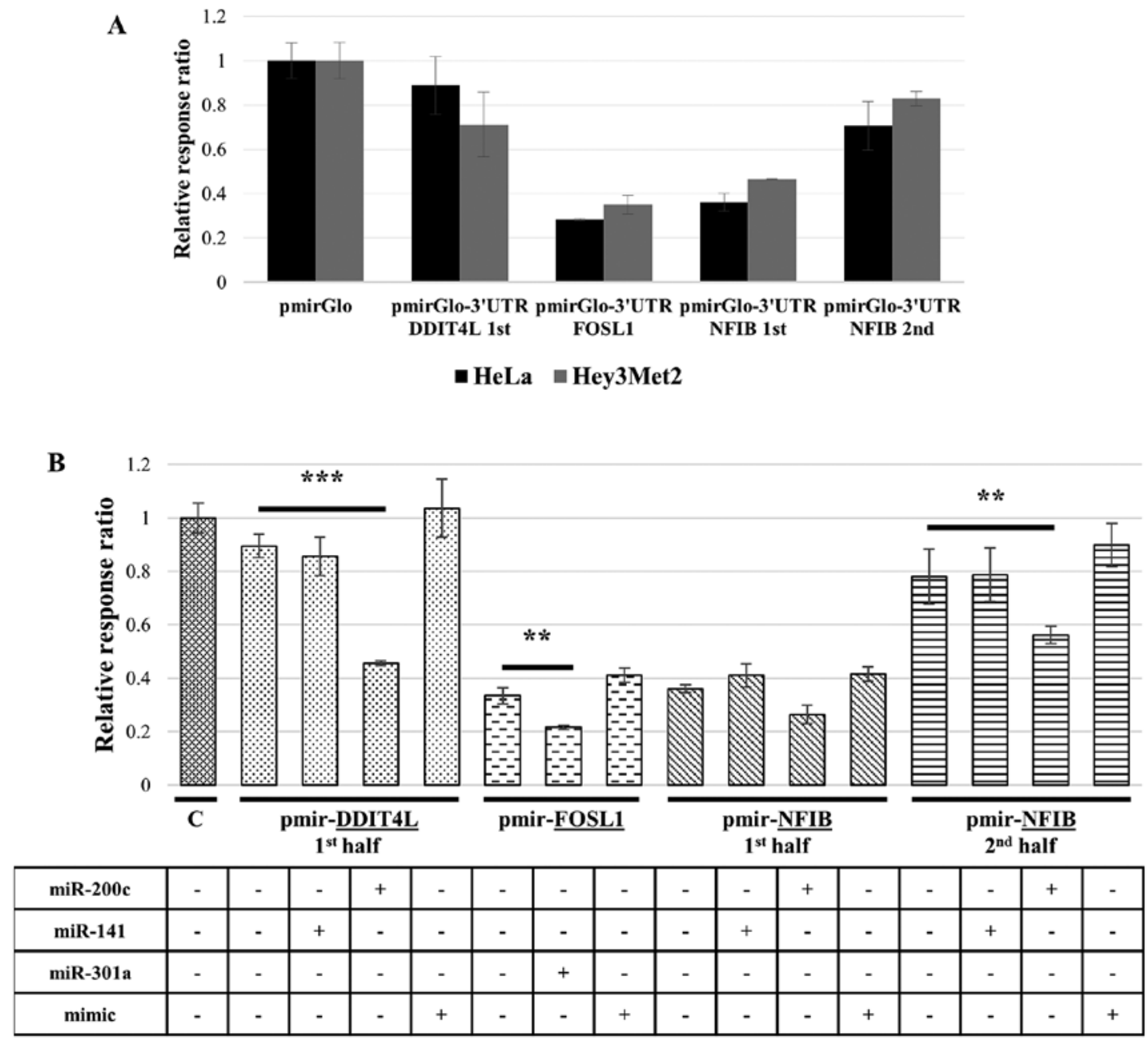

Figure 3. Luciferase reporter gene assay on two cell lines known to express low levels of endogenous RNASET2. (A) The 3'UTRs regions of the selected target genes (DDIT4L, FOSL1 and NFIB, the latter split into two subfrangments named 1st and 2nd) were cloned in pmirGLO reporter vector (Promega) and the recombinant constructs were transiently transfected in HeLa and Hey3Met2 cells. After the addiction of Dual-Glo substrate, firefly and Renilla luciferase activities were measured. Results are calculated as ratio of firefly:Renilla luminescence and normalized to the ratio from empty vector wells. Error bars represent standard error of the mean. (B) The same recombinant vectors used for the experiment shown in (A) were co-transfected with the corresponding miRNAs in HeLa cells to confirm the activity of these miRNAs on the corresponding 3'UTRs. Firefly and Renilla luciferase activities were measured as previously described. Results are calculated as ratio of firefly:Renilla luminescence and normalized to the ratio from empty vector wells. Error bars represent standard error of the mean. Statistical analysis was performed using two-tailed Student's t-test. ${ }^{* * *} \mathrm{P}<0.01 ;{ }^{* * *} \mathrm{P}<0.001$.

we could not include this gene in subsequent investigations. Due to the same problem, only the first half from the $3^{\prime} \mathrm{UTR}$ region of the DDIT4L gene could be cloned in pmirGLO. Finally, due to its large size (6487 bp), the 3'UTR regions from the NFIB gene was split into two sub-fragments that were independently cloned into the reporter vector.

Since parental OVCAR3 cells express high levels of endogenous RNASET2 protein (13), the recombinant reporter constructs carrying the different 3'UTR regions were first transfected in two cell lines (HeLa and Hey3Met2) known to express low levels of endogenous RNASET2. A subsequent luciferase gene-based assay showed a high expression level of the luciferase gene (similar to those observed for the control pmirGLO empty vector) in both cell lines for reporter constructs carrying the DDIT4L 3'UTR cloned subregion and the second half of the NFIB $3^{\prime}$ UTR, suggesting that in the presence of very low RNASET2 expression the selected 3'UTR regions of the endogenous transcripts from these genes do not undergo miRNA-mediated regulation in either cell line
(Fig. 3A). By contrast, a partial repression of reporter gene expression was observed following transfection of the 3'UTR regions from the FOSL1 and the first half of the NFIB 3'UTR regions only, suggesting that putative endogenous miRNAs different from those selected in this study might possibly target some of the relevant 3'UTR regions in these two cell lines.

To verify that the selected miRNAs were indeed capable of repressing these putative target genes via binding to their 3'UTR regions, the same reporter constructs were co-transfected in HeLa cells together with the corresponding miRNAs, and luciferase expression assays were again carried out. Significantly, a clear effect was found for $m i R-200 c$ on reporter gene expression driven by recombinant constructs carrying DDIT4L (first 3'UTR half) and NFIB (second 3'UTR half) regions (Fig. 3B). A less pronounced, but detectable effect on reporter gene expression was observed for $m i R-200 c$ on the $N F I B$-carrying construct (first 3'UTR half) and for $m i R-301 a$ on the FOSL1-carrying construct (Fig. 3B). The observed effects were shown to be specific for the selected 


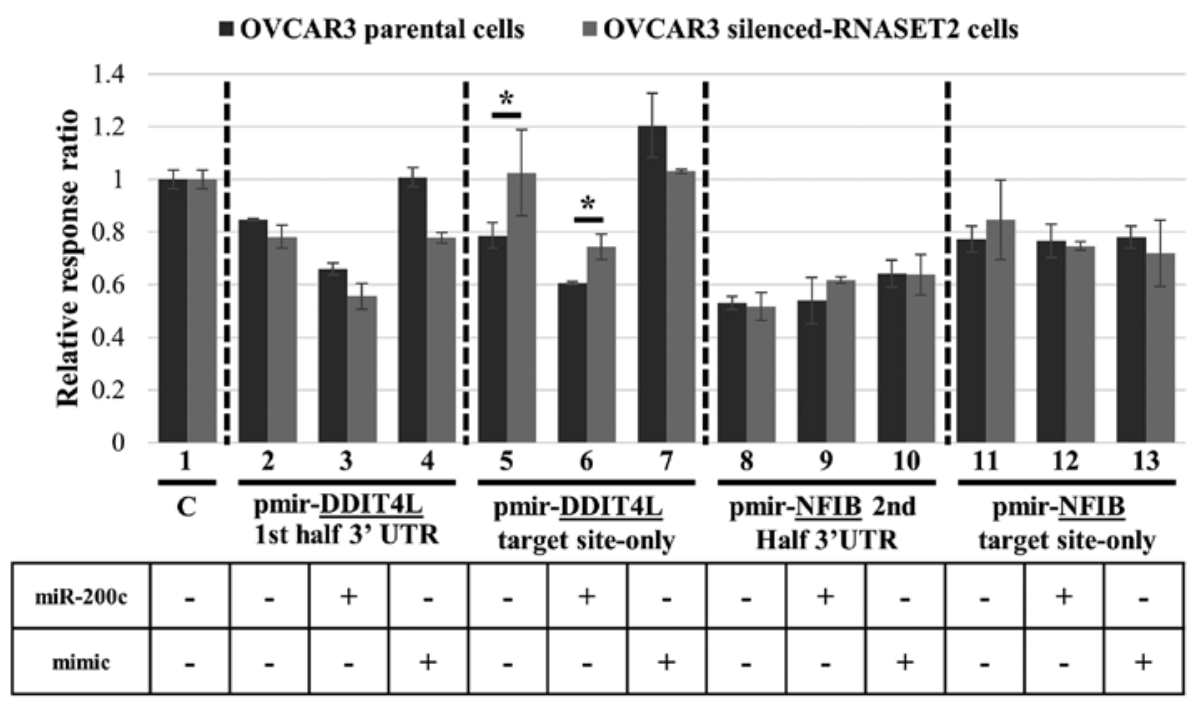

Figure 4. Luciferase reporter gene assays on OVCAR3 cell transfectants. Control OVCAR3 cells and RNASET2-silenced cells were transfected with the recombinant constructs carrying either the whole 3'UTR regions ('whole UTR' in the table) or a smaller region representing just the predicted miR-200c target sequence within the 3'UTRs ('target site-only' in the table) from the DDIT4L gene or the second half of the 3'UTR region from the NFIB gene, with and without the corresponding miRNAs. After addiction of Dual-Glo substrate, firefly and Renilla luciferase activities were measured. Results were calculated from triplicate reads as ratio of firefly:Renilla luminescence and normalized to the ratio from pmirGLO empty vector wells. Error bars represent standard error of the mean. Statistical analysis was performed using two-tailed Student's t-test. ${ }^{*} \mathrm{P}<0.05$.

miRNAs, since transfection with unrelated miRNAs for which no binding sites were present in the 3'UTR regions under investigation ('mimic' miRNAs) had no effect on luciferase expression. Taken together, these data confirmed that some of the downregulated miRNAs in RNASET2-silenced cells are actually able to repress the expression of potential target mRNAs by means of interaction with the corresponding 3'UTR regions.

To further verify the role of RNASET2 in miRNA-mediated regulation of these potential target genes, we turned back to our OVCAR3 experimental model. The 3'UTR-luciferase reporter plasmids from the two genes shown to be strongly repressed by miR-200c (DDIT4L-first 3'UTR half and NFIB-second 3'UTR half) were transiently transfected in RNASET2-silenced (shT2) and control OVCAR3 cells. In order to avoid putative nonspecific effects from unrelated endogenous miRNAs, for both genes we used pmirGLO reporter constructs bearing either the whole 3'UTR region under investigation or a shorter DNA sequence representing just the predicted $m i R-200 c$ target sequence within the 3'UTRs (labeled 'target site-only').

Strikingly, the RNASET2 expression status was indeed associated with the level of luciferase expression when the reporter vector bearing just the $m i R-200 c$ target site in the 3'UTR of DDIT4L was used, since expression of the luciferase reporter was increased in RNASET2-silenced cells, as expected (Fig. 4, lane 5). Moreover, co-transfection of $m i R$ $200 c$ in both control and RNASET2-silenced cells led to a decrease in reporter expression, again supporting a role for $m i R-200 c$ in targeting the $3^{\prime} \mathrm{UTR}$ region of DDIT4L (Fig. 4, lane 6). By contrast, the reporter vectors carrying the whole DDIT4L 3'UTR did not show a significant effect (Fig. 4, lanes 2 and 3). Once again, dowregulation of luciferase by miR-200c through DDIT4L 3'UTR binding was shown to be specific, since a mimic miRNA did not trigger a decrease in reporter gene expression with respect to the control (Fig. 4, lane 7). These results are in agreement with the notion that miR-200c-mediated repression of DDIT4L is somehow impaired following RNASET2-dowregulation in OVCAR3 cells. By contrast, no effect of RNASET2 expression status was observed following transfection of recombinant constructs bearing the 3'UTR of NFIB in both forms ('2nd half 3'UTR' and 'target site-only') (Fig. 4, lanes 8, 9, 11 and 12).

Since the gene expression profile data were derived from pools of transfected OVCAR3 cells, to further validate these results we performed the same assay in several independent OVCAR3 clones that were not used in the microarray analysis. Again, an inverse correlation between RNASET2 expression status and reporter gene expression was observed when the 'target site-only' construct for DDIT4L 3'UTR region was used (data not shown). Moreover, a slight effect was observed also for NFIB (second 3'UTR half) in this experiment (data not shown). Altogether, the data show a consistent trend for a role of RNASET2 expression status on miR-200c-mediated silencing of DDIT4L.

The DDIT4L gene (also known as REDD2 or RTP801L) is involved in the cell growth control by regulating the mTOR signaling pathway (21). Since expression of this gene turned out to be affected in RNASET2-silenced OVCAR3 cells, likely via mir-200c downregulation, we wondered whether RNASET2 silencing in OVCAR3 cells might be associated with a change in cell size due to DDIT4L upregulation. Notably, following fluorescent staining of OVCAR3 cells with a marker for the actin cytoskeleton (phalloidin-TRITC) a clear change in average cell size in RNASET2-silenced OVCAR3 cells was observed with respect to control cells (Fig. 5A and B), suggesting that RNASET2-mediated increase in DDIT4L expression in these cells might indeed affect cell growth. To further validate the involvement of DDIT4L in the observed RNASET2-mediated changes in cell size, the activation state of the MTOR signaling pathway was compared in control and RNASET2-silenced 


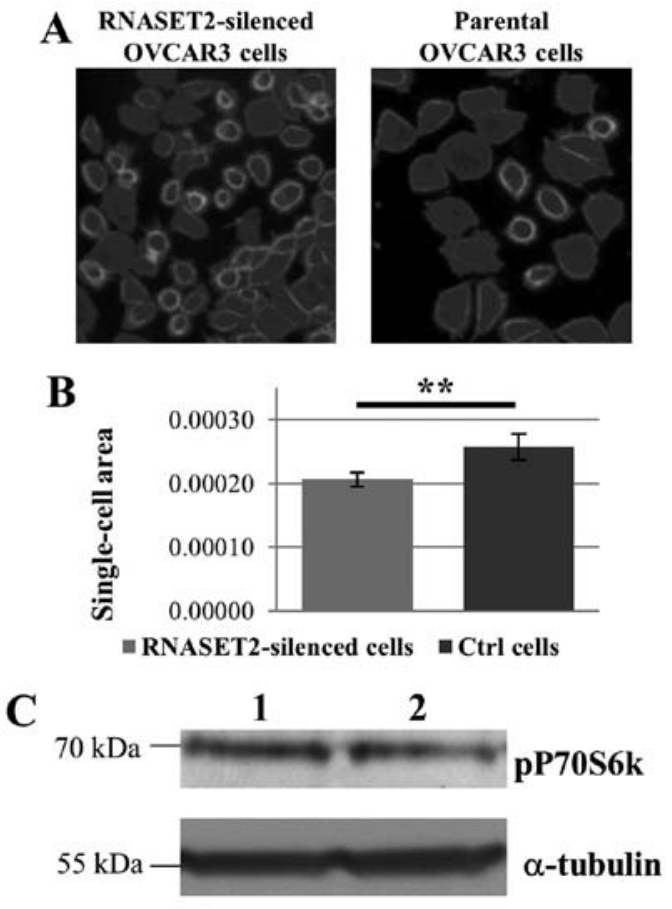

Figure 5. Silencing of RNASET2 expression affects cell size and mTOR pathway in OVCAR3 cells. (A) OVCAR3 cell clones were seeded on coverslips and stained with TRITC-conjugated phalloidin. (B) Cell size analysis was performed using ImageJ software. A slight reduction in average cell size is observed in RNASET2-silenced OVCAR 3 cells. ${ }^{* *} \mathrm{P}<0.01$ statistically significant values analyzed by the Student's t-test. (C) Western blot analysis for p70S6K expression in parental (lane 1) and RNASET2-silenced (lane 2) OVCAR3 cells. P70S6K, 70-kDa ribosomal protein S6 kinase.

OVCAR3 cells by evaluating the phosphorylation status of the mTOR downstream effector S6K1 kinase. As shown in Fig. 5C, phosphorylation of S6K1 turned out to be slightly decreased in OVCAR3 cells following RNASET2 silencing, thus, lending support to the notion that RNASET2-mediated changes in the transcript levels of DDIT4L gene might affect the activation state of mTOR pathway.

\section{Discussion}

The human RNASET2 gene has been recently added to the growing list of ribonuclease-encoding genes endowed with a tumor suppressor activity (22). Indeed, detailed investigations on the role played by RNASET2 in vivo based on murine xenograft models unveiled a strong non-cell autonomous oncosuppressive role for this gene in two independent human ovarian cancer models $(12,13)$. Remarkably, the observed tumor suppressive role of RNASET2 was linked to the stimulation of a marked innate immune response involving cells of the monocyte-macrophage lineage and was shown to be independent of the enzyme catalytic activity (23). On the other hand, several biological functions of T2 family members are dependent on their ability to process or degrade RNA substrates, and some of these functions might be related to tumor suppression as well (22). In this context, it is likely that the putative catalysisdependent role(s) of RNASET2 are carried out within the same cells expressing it and, as such, they could reveal themselves in a cell-autonomous manner. Indeed, a recent study carried out in the same experimental model (the OVCAR3 human ovarian cancer cell line) used to report the non-cell autonomous oncosuppressive role of RNASET2 allowed us to show that this gene can also affect several cancer-related parameters in a cell-autonomous manner (14). Significantly, RNASET2 was shown to call into play these cell-autonomous roles in response to a wide range of cellular stresses, some of which are known to be frequently observed in both pre-cancerous lesions and advanced cancer (14). Moreover, the recent discovery that the RNASET2 protein co-localizes to P-bodies under stress conditions strongly suggests that the stress response role played by this enzyme might at least in part rely on its catalytic activity, since P-bodies are widely known to represent intracellular sites of RNA processing and degradation (19).

Building on this evidence, in the present study we addressed the effects of RNASET2 silencing on the miRNA transcriptome, since the latter is known to come into play in P-bodies. Indeed, RNASET2 silencing in OVCAR3 cells was shown to significantly affect the expression level of several miRNAs, some of which have been previously involved in ovarian cancer pathogenesis $(3,20)$. Moreover, the two miRNAs showing the strongest impact on the gene expression profile of RNASET2silenced OVCAR3 cells ( $m i R-200 c$ and $m i R-141$; Fig. 1) have been reported to be disregulated in several human cancers, including ovarian carcinoma $(24,25)$, thus, lending support to the hypothesis that the observed tumor suppressive role played by RNASET 2 might at least in part be carried out by means of miRNA genes deregulation. To the best of our knowledge, this is the first report on the effects of a mammalian T2 RNase family member on the miRNA transcriptome. Moreover, by addressing the mRNA profile in the same cells we found a much more extended effect of RNASET2 silencing on the global cell transcriptome, with approximately 300 transcript being affected. Taken together, these data suggest that the expression levels of RNASET2 seem to have a deep impact on the cell's global transcriptome in our experimental model.

Contrary to our expectations, a great proportion of transcripts (both miRNAs and mRNAs) turned out to be downregulated rather than upregulated in the absence of RNASET2, thus, suggesting that the mechanism(s) by which this ribonuclease affects the expression level of several intracellular transcripts might not involve a direct cleavage of target RNAs. Of note, in a previous report microarray-based gene expression profiling of the Hey3Met2 ovarian cancer cell line showed that overexpression of a catalytically-dead RNASET2 protein could nevertheless affect the cell transcriptome significantly, suggesting that this protein can affect the cellular gene expression profile independently of its ribonucleolytic activity (26). These data are in keeping with several recent reports showing that proteins belonging to the T2 family members can affect several biological processes in a catalytically-independent manner (22).

Although miRNAs are known to individually regulate several independent mRNAs, evidence for a miRNA-mediated mechanism of gene regulation could not be found for most transcripts whose expression was dependent on the RNASET2 expression status. However, among the mRNAs whose expression levels where changed in RNASET2-silenced cells, a handful was shown to represent potential target genes for a few ovarian-cancer related, RNASET2-sensitive miRNAs, thus, 
suggesting that regulation of these mRNAs by RNASET2 might involve changes in the expression levels of their cognate miRNAs.

In support of this hypothesis, we found that two candidate miRNAs ( $m i R-200 c$ and $m i R-301 a)$ were indeed able to affect reporter gene expression levels when the 3'UTR regions of the corresponding candidate target genes (DDIT4L, NFIB and FOSL1) were cloned downstream of a luciferase reporter construct. The role of RNASET2 in miRNA-mediated silencing of one of these genes (DDIT4L) was further suggested by showing an inverse correlation between the expression levels of RNASET2 and the luciferase gene when the corresponding reporter constructs were assessed in OVCAR3 cells. Finally, since one of the candidate RNASET2 target genes (DDIT4L, whose expression was shown to be regulated by $m i R-200 c$ ) is functionally involved in the regulation of the mTOR pathway (21) the effect of RNASET2 expression levels on cell growth (a cell phenotype related to cancer and modulated by this pathway) was evaluated in our experimental model. Indeed, RNASET 2 expression levels in OVCAR3 cells were shown to affect two parameters downstream of DDIT4L, i.e. cell size and the activation of the mTOR pathway. In this regard it is worth noting that, whereas a slight decrease in mTOR activation pathway was observed in relation to RNASET2 expression levels, a much more evident effect was observed on cell size. We considered that such discrepancy might be attributed to the established pleiotropic roles of the RNASET2 protein, which has been previously shown to significantly affect cell shape in OVCAR3 cells by remodeling the actin cytoskeleton (14).

Of note, RNASET2 expression has been previously shown to be increased in several cell lines (including OVCAR3 cells) under hypoxic conditions (14) and expression of DDIT4L is known to be HIF-dependent (27) suggesting that, under hypoxic conditions, upregulation of RNASET2 expression might affect cell growth in part via DDIT4L. Further investigations will be focused on this topic. Of note, a cancer cell-related parameter (cell proliferation rate) has been recently shown to be affected by RNASET 2 expression levels in OVCAR3 cells under hypoxic conditions (14).

Though preliminary, these results provide to the best of our knowledge the first evidence that a member of the T2 RNAse family might affect the expression pattern of selected miRNAs in mammalian cells. The reason why, among the many miRNAs potentially expressed in ovarian cancer cells, only a few are actually affected by RNASET 2 silencing has not been addressed in this study and will require further investigations. To date, $\mathrm{T} 2$ ribonucleases have been described as rather aspecific ribonucleolytic enzymes and only a few specific targets (such as rRNA) have been reported for this class of RNases (28). Therefore, although targeting of RNASET2 to P-bodies might suggest a rather general role in stress-induced RNA processing for this RNase, the results of this study suggest the occurrence of a sort of selectivity in target selection by this protein, although the mechanism ruling such putative selectivity are largely unknown. Our results thus, further confirm that the effects of RNASET2 on the cell transcriptome are more complex than expected, as already observed in previous investigations on the catalytically-dead RNASET2 protein (26).
Finally, although some members of the $m i R-200$ gene family that were found to be downregulated in RNASET2-silenced cells have been reported by some authors to be upregulated in advanced ovarian cancer samples $(29,30)$, it should be acknowledged that the role played by the $m i R-200$ gene family in cancer pathogenesis is still rather controversial. For instance, high expression of $m i R-200 c$ was correlated with a decreased risk of disease recurrence in patients with serous ovarian carcinoma (31) and re-expression of $m i R-200 \mathrm{c}$ in aggressive ovarian cancer cell lines was shown to trigger a wide range of oncosuppressive effects both in vitro and in vivo $(32,33)$. Furthermore, $m i R-200 c$ was reported to be downregulated in a subpopulation of cell expressing the cancer stem cell marker CD133 in the same ovarian cancer cell line (OVCAR3) used in this study (34). Therefore, it is likely that the dowregulation of $m i R-200 c$ observed in RNASET2-silenced OVCAR3 cells might contribute to the cell-autonomous oncosuppressive role ascribed to this gene.

In conclusion, the present study shed further light on the pleiotropic roles played by the human RNASET 2 gene in the context of its oncosuppressive role. The accumulating evidence suggesting the role of RNASET2 as a stress-inducible gene, which orchestrates a tumor suppressive response in a both cell-autonomous and non-cell autonomous manners, is compatible with a model whereby increased expression of this gene might represent a key tool in mammalian anticancer response. Our results suggest that this response might in part entail the regulation of cancer-related genes by means of RNASET2-mediated modulation of miRNA expression.

\section{Acknowledgements}

The present study is in memory of our friend and colleague Giovanna Turconi, who is no longer with us. We all miss her so much. We are also very grateful to Giovanna's relatives and friends for their support to the present work. Marco Fabbri is a student of the PhD program in Biotechnology, School of Biological and Medical Sciences, University of Insubria (Italy). Francesco Acquati was supported by Giovanna Turconi's Memorial Funds.

\section{References}

1. Siegel R, Naishadham D and Jemal A: Cancer statistics, 2012. CA Cancer J Clin 62: 10-29, 2012.

2. Sopik V, Iqbal J, Rosen B and Narod SA: Why have ovarian cancer mortality rates declined? Part I. Incidence. Gynecol Oncol 138: 741-749, 2015.

3. Dahiya N and Morin PJ: MicroRNAs in ovarian carcinomas. Endocr Relat Cancer 17: F77-F89, 2010

4. Li SD, Zhang JR, Wang YQ and Wan XP: The role of microRNAs in ovarian cancer initiation and progression. J Cell Mol Med 14: 2240-2249, 2010.

5. Katz B, Tropé CG, Reich R and Davidson B: MicroRNAs in Ovarian Cancer. Hum Pathol 46: 1245-1256, 2015.

6. Bartel DP: MicroRNAs: Genomics, biogenesis, mechanism, and function. Cell 116: 281-297, 2004.

7. Hwang HW and Mendell JT: MicroRNAs in cell proliferation, cell death, and tumorigenesis. Br J Cancer 94: 776-780, 2006

8. Esquela-Kerscher A and Slack FJ: Oncomirs - microRNAs with a role in cancer. Nat Rev Cancer 6: 259-269, 2006.

9. Acquati F, Morelli C, Cinquetti R, Bianchi MG, Porrini D, Varesco L, Gismondi V, Rocchetti R, Talevi S, Possati L, et al: Cloning and characterization of a senescence inducing and class II tumor suppressor gene in ovarian carcinoma at chromosome region 6q27. Oncogene 20: 980-988, 2001. 
10. Monti L, Rodolfo M, Lo Russo G, Noonan D, Acquati F and Taramelli R: RNASET2 as a tumor antagonizing gene in a melanoma cancer model. Oncol Res 17: 69-74, 2008.

11. Smirnff P, Roiz L, Algelkovitch B, Schwartz B and Shoseyov O: A recombinant human RNASET2 glycoprotein with antituorigenic and antiangiogenic characteristics. Cancer 107: 2760-2769, 2006.

12. Acquati F, Bertilaccio S, Grimaldi A, Monti L, Cinquetti R, Bonetti P, Lualdi M, Vidalino L, Fabbri M, Sacco MG, et al: Microenvironmental control of malignancy exerted by RNASET2, a widely conserved extracellular RNase. Proc Natl Acad Sci USA 108: 1104-1109, 2011.

13. Acquati F, Lualdi M, Bertilaccio S, Monti L, Turconi G, Fabbri M, Grimaldi A, Anselmo A, Inforzato A, Collotta A, et al: Loss of function of Ribonuclease T2, an ancient and phylogenetically conserved RNase, plays a crucial role in ovarian tumorigenesis. Proc Natl Acad Sci USA 110: 8140-8145, 2013.

14. Lualdi M,Pedrini E, Rea K, Monti L, Scaldaferri D, Gariboldi M, Camporeale A, Ghia P, Monti E, Tomassetti A, et al: Pleiotropic modes of action in tumor cells of RNASET2, an evolutionary highly conserved extracellular RNase. Oncotarget 6: 7851-7865, 2015.

15. Huber W, Carey VJ, Gentleman R, Anders S, Carlson M, Carvalho BS, Bravo HC, Davis S, Gatto L, Girke T, et al: Orchestrating high-throughput genomic analysis with bioconductor. Nat Methods 12: 115-121, 2015.

16. Huang W, Sherman BT and Lempicki RA: Systematic and integrative analysis of large gene lists using DAVID bioinformatics resources. Nat Protoc 4: 44-57, 2009.

17. Livak KJ and Schmittgen TD: Analysis of relative gene expression data using real-time quantitative PCR and the 2(-Delta Delta C(T)) method. Methods 25: 402-408, 2001.

18. Vidalino L, Monti L, Haase A, Moro A, Acquati F, Taramelli R and Macchi P: Intracellular trafficking of RNASET2, a novel component of P-bodies. Biol Cell 104: 13-21, 2012.

19. Eulalio A, Behm-Ansmant I and Izaurralde E: P bodies: At the crossroads of post-transcriptional pathways. Nat Rev Mol Cell Biol 8: 9-22, 2007.

20. Wang L, Zhu MJ, Ren AM, Wu HF, Han WM, Tan RY and Tu RQ: A ten-microRNA signature identified from a genomewide microRNA expression profiling in human epithelial ovarian cancer. PLoS One 9: e96472, 2014.

21. Corradetti MN, Inoki K and Guan KL: The stress-inducted proteins RTP801 and RTP801L are negative regulators of the mammalian target of rapamycin pathway. J Biol Chem 280: 9769-9772, 2005.

22. Luhtala N and Parker R: T2 Family ribonucleases: Ancient enzymes with diverse roles. Trends Biochem Sci 35: 253-259, 2010 .
23. Acquati F, Possati L, Ferrante L, Campomenosi P, Talevi S, Bardelli S, Margiotta C, Russo A, Bortoletto E, Rocchetti R, et al: Tumor and metastasis suppression by the human RNASET2 gene. Int J Oncol 26: 1159-1168, 2005.

24. Kumar S, Nag A and Mandal CC: A comprehensive review on miR-200c, a promising cancer biomarker with therapeutic potential. Curr Drug Targets 16: 1381-1403, 2015.

25. Li XY, Li H, Bu J, Xiong L, Guo HB, Liu LH and Xiao T: Prognostic role of microRNA-200c-141 cluster in various human solid malignant neoplasms. Dis Markers 2015: 935626, 2015.

26. Acquati F, Monti L, Lualdi M, Fabbri M, Sacco MG, Gribaldo L and Taramelli R: Molecular signature induced by RNASET2, a tumor antagonizing gene, in ovarian cancer cells. Oncotarget 2: 477-484, 2011.

27. Cuaz-Pérolin C,Furman C, Larigauderie G, Legedz L, Lasselin C, Copin C, Jaye M, Searfoss G, Yu KT, Duverger N, et al: REDD2 gene is upregulated by modified LDL or hypoxia and mediates human macrophage cell death. Arterioscler Thromb Vasc Biol 24: 1830-1835, 2004.

28. Haud N, Kara F, Diekmann S, Henneke M, Willer JR, Hillwig MS, Gregg RG, Macintosh GC, Gärtner J, Alia A, et al: rnaset2 mutant zebrafish model familial cystic leukoencephalopathy and reveal a role for RNase T2 in degrading ribosomal RNA. Proc Natl Acad Sci USA 108: 1099-1103, 2011.

29. Nam EJ, Yoon H, Kim SW, Kim H, Kim YT, Kim JH, Kim JW and Kim S: MicroRNA expression profiles in serous ovarian carcinoma. Clin Cancer Res 14: 2690-2695, 2008.

30. Chen Y, Zhang L and Hao Q: Candidate microRNA biomarkers in human epithelial ovarian cancer: Systematic review profiling studies and experimental validation. Cancer Cell Int 13: 86-94, 2013.

31. Leskelä S, Leandro-García LJ, Mendiola M, Barriuso J, Inglada-Pérez L, Muñoz I, Martínez-Delgado B, Redondo A, de Santiago J, Robledo M, et al: The miR-200 family controls beta-tubulin III expression and is associated with paclitaxelbased treatment response and progression-free survival in ovarian cancer patients. Endocr Relat Cancer 18: 85-95, 2010.

32. Cochrane DR, Howe EN, Spoelstra NS and Richer JK: Loss of miR-200c: A marker of aggressiveness and chemoresistance in female reproductive cancers. J Oncol 2010: 821717, 2010.

33. Chen D, Zhang Y, Wang J, Chen J, Yang C, Cai K, Wang X, Shi F and Dou J: MicroRNA-200c overexpression inhibits tumorigenicity and metastasis of $\mathrm{CD} 117^{+} \mathrm{CD} 44^{+}$ovarian cancer stem cells by regulating epithelial-mesenchymal transition. J Ovarian Res 6: 50-60, 2013

34. Guo R, Wu Q, Liu F and Wang Y: Description of the CD133+ subpopulation of the human ovarian cancer cell line OVCAR3. Oncol Rep 25: 141-146, 2011. 